\title{
Selective modulation of TLR4-activated inflammatory responses by altered iron homeostasis in mice
}

\author{
Lijian Wang, ${ }^{1,2}$ Lynne Harrington, ${ }^{1}$ Estela Trebicka, ${ }^{1}$ Hai Ning Shi, ${ }^{1,3}$ Jonathan C. Kagan, ${ }^{4}$ \\ Charles C. Hong, ${ }^{5}$ Herbert Y. Lin, ${ }^{6}$ Jodie L. Babitt, ${ }^{6}$ and Bobby J. Cherayil $1{ }^{1,3}$ \\ ${ }^{1}$ Mucosal Immunology Laboratory, Massachusetts General Hospital, Charlestown, Massachusetts, USA. 2Department of Nutrition, \\ Harvard School of Public Health, Boston, Massachusetts, USA. ${ }^{3}$ Department of Pediatrics, Harvard Medical School, Boston, Massachusetts, USA. \\ ${ }^{4}$ Department of Gastroenterology, Children's Hospital Boston, Boston, Massachusetts, USA. ${ }^{5}$ Division of Cardiovascular Medicine, \\ Vanderbilt University Medical Center, Nashville, Tennessee, USA. ${ }^{6}$ Program in Membrane Biology, \\ Division of Nephrology and Center for Systems Biology, Massachusetts General Hospital, Boston, Massachusetts, USA.
}

\begin{abstract}
Mice deficient in the hemochromatosis gene, $\mathrm{Hfe}$, have attenuated inflammatory responses to Salmonella infection associated with decreased macrophage TNF- $\alpha$ and IL-6 biosynthesis after exposure to LPS. In this study, we show that the abnormal cytokine production is related to impaired TLR4 signaling. Despite their abnormal response to LPS, Hfe KO macrophages produced amounts of TNF- $\alpha$ similar to those in WT cells after TLR2 stimulation. Consistent with this finding, LPS-induced activation of Mal/MyD88-dependent events was normal in the mutant macrophages. However, LPS-induced IFN- $\beta$ expression, a TRAM/TRIF-dependent response activated by TLR4, was reduced by $H f e$ deficiency. This reduction could be replicated in WT macrophages with the use of iron chelators. In contrast, TLR3-activated expression of IFN- $\beta$, a TRIF-dependent response, was normal in $\mathrm{Hfe} \mathrm{KO}$ macrophages and was unaffected by iron chelation. Our data suggest that low intracellular iron selectively impairs signaling via the TLR4/TRAM/TRIF pathway proximal to TRIF and results in reduced LPS-induced cytokine expression. Furthermore, by mimicking the altered iron metabolism associated with $\mathrm{Hfe}$ deficiency, we found that 3 different inhibitors of hepcidin attenuated Salmonella-induced and noninfectious enterocolitis. Thus, manipulation of iron homeostasis could represent a new therapeutic approach to controlling inflammation.
\end{abstract}

\section{Introduction}

Iron is an essential micronutrient for both microbial pathogens and their mammalian hosts (1). Consequently, disordered iron homeostasis in humans and experimental animal models is associated with alterations in the course of infectious disease. Conditions of iron overload have been shown to predispose to infections such as salmonellosis and tuberculosis (2-6). Similarly, iron deficiency confers relative resistance to infection, whereas iron supplements can reverse this effect (7-10). The effects of altered iron metabolism on infectious disease have been attributed to changes in the host immune response, as well as direct effects of iron on microbial growth $(1,11)$, but the underlying mechanisms remain poorly understood.

Hereditary hemochromatosis is a genetically determined iron overload disease (12). The most common form, type I, is associated with variations in the hemochromatosis gene, $H F E$, which encodes an atypical class I MHC protein expressed on hepatocytes, macrophages, and intestinal crypt cells (13). The HFE protein is involved in sensing circulating iron status and initiating signals that regulate the expression of hepcidin, a secreted hepatocyte peptide that plays a key role in iron homeostasis (14-16). Hepcidin expression is upregulated by iron loading and inflammation, whereas it is inhibited by iron deficiency, anemia, and hypoxia $(14,15)$. It binds

Conflict of interest: H.Y. Lin and J.L. Babitt have ownership interest in Ferrumax Pharmaceuticals, which has licensed technology from Massachusetts General Hospital based on their work. A patent application entitled "Methods and Composition to Regulate Iron Metabolism" has been submitted by Massachusetts General Hospital. Citation for this article: J. Clin. Invest. 119:3322-3328 (2009). doi:10.1172/JCI39939. to the iron exporter ferroportin (FPN), expressed on the surface of macrophages and duodenal enterocytes, and promotes lysosomal degradation of this protein (17). Hepcidin-induced downregulation of FPN thus inhibits cellular iron export from the intestinal epithelium and macrophages. The HFE/hepcidin/FPN axis is a major regulatory mechanism that maintains iron homeostasis in response to changing requirements. When HFE fails to function normally, FPN expression is elevated because of low circulating hepcidin levels; the resulting increase in iron absorption from the gut and release from phagocytes leads to pathologic deposition of the metal in various tissues (12). Mice with homozygous disruption of the Hfe gene recapitulate several features of type I hemochromatosis, including low hepcidin, elevated macrophage FPN, and high serum and liver iron levels (18-21).

Type I hemochromatosis is associated with increased susceptibility to pathogens such as Yersinia enterocolitica and Vibrio vulnificus (22-24). In keeping with these clinical observations, we found recently that mice deficient in $\mathrm{Hfe}$ have an abnormal innate immune response to oral infection with Salmonella typhimurium, characterized by attenuated intestinal inflammation and increased tissue pathogen burden (25). Investigation of the underlying mechanism revealed that peritoneal macrophages from the mutant animals had decreased intracellular free iron levels, consistent with elevated FPN expression. Moreover, the low intracellular iron had an inhibitory effect on Salmonella- and LPS-induced upregulation of the proinflammatory cytokines TNF- $\alpha$ and IL-6. In the present work, we elucidate how the abnormality of iron metabolism caused by $H f e$ deficiency leads to impaired innate immune responses. We 


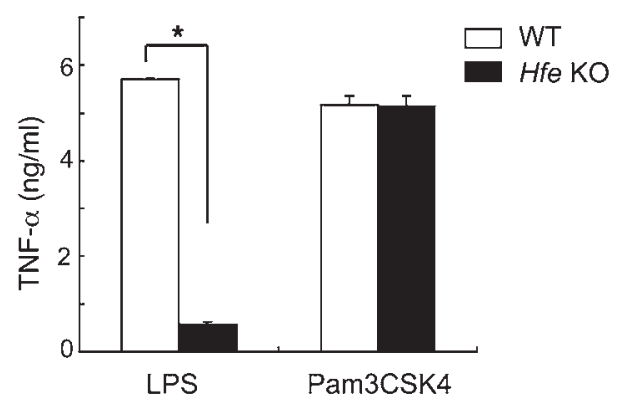

\section{Figure 1}

Hfe KO macrophages have an abnormal cytokine response to stimulation with TLR4, but not TLR2. Peritoneal macrophages from WT and Hfe KO mice were stimulated with $100 \mathrm{ng} / \mathrm{ml}$ LPS $(n=4)$ or $1 \mu \mathrm{g} / \mathrm{ml}$ Pam3CSK4 $(n=3)$ for 6 hours. Supernatants were collected and analyzed by ELISA for TNF- $\alpha$. Data represent mean \pm SD. Similar results were obtained in 2 separate experiments. ${ }^{*} P<0.0001$.

also show that manipulation of iron homeostasis may represent a novel approach to controlling inflammation.

\section{Results}

Abnormal inflammatory cytokine expression in $\mathrm{Hfe} \mathrm{KO}$ macrophages is associated with intact Mal/MyD88-dependent responses. We previously showed that production of the proinflammatory cytokines TNF- $\alpha$ and IL- 6 was significantly reduced in $\mathrm{Hfe} \mathrm{KO}$ peritoneal macrophages after Salmonella infection or LPS treatment (25), which indicates an abnormality in the response to TLR 4 activation. To investigate the underlying mechanism, we examined responses to activation of other TLRs. In contrast to the significant reduction in LPS-induced TNF- $\alpha$ expression in the Hfe KO macrophages, we found that the response to TLR2 activation with the synthetic lipopeptide ligand Pam3CSK4 was normal in the mutant cells (Figure 1). In our previous work, we presented evidence indicating that the abnormal response to TLR4 activation was related to the low intracellular iron levels in the Hfe KO macrophages. To further substantiate this idea, we used an assay based on quenching of calcein fluorescence by free iron (25) to evaluate peritoneal macrophages immediately after harvest and after overnight culture in vitro. Freshly isolated $\mathrm{Hfe} \mathrm{KO}$ macrophages had appreciably higher calcein fluorescence than did WT cells (Supplemental Figure 1; supplemental material available online with this article; doi:10.1172/JCI39939DS1), indicative of lower intracellular iron levels and consistent with our previously published observations (25). After overnight incubation, however, the calcein fluorescence of the Hfe KO macrophages decreased to the level of the WT cells (Supplemental Figure 1), which indicates that the conditions of prolonged in vitro culture resulted in normalization of intracellular iron in the Hfe $\mathrm{KO}$ cells. Moreover, after overnight culture, there was no longer any difference between the WT and mutant macrophages with respect to LPS-induced TNF- $\alpha$ production (Supplemental Figure 2; compare with Figure 1). Overnight treatment of the macrophages with hepcidin at a dose that has previously been shown to downregulate FPN expression and elevate intracellular iron (17) led to a significant increase in the amount of TNF- $\alpha$ produced in response to LPS by both WT and Hfe KO macrophages (Supplemental Figure 2), a finding consistent with our previously reported observation that iron loading of WT macrophages leads to increased LPS-induced TNF- $\alpha$ expression (25). Finally, we found that, unlike freshly isolated peritoneal macrophages, there was no difference in LPS-induced TNF- $\alpha$ production between WT and Hfe KO bone marrow-derived macrophages (Supplemental Figure 3; compare with Figure 1). Thus, multiple lines of evidence support the notion that the abnormal TLR4-activated cytokine response of the Hfe $\mathrm{KO}$ macrophages is caused by the low intracellular iron in these cells rather than being a cell-autonomous consequence of $H f e$ deficiency. It should be noted that although we present herein only the observations on TNF- $\alpha$ expression, similar findings were obtained when IL-6 production was analyzed (data not shown).

TLR4 is connected to 2 major signal transduction pathways via 2 sets of adaptor proteins - myeloid differentiation primary response protein 88 (MyD88) and MyD88-adaptor like (Mal) or Toll/IL-1 receptor domain-containing adaptor inducing IFN- $\beta$ (TRIF) and TRIF-related adaptor molecule (TRAM) - with each contributing to different cellular responses, whereas TLR2 activates only the Mal/MyD88 pathway $(26,27)$. Our observation that the cytokine response to TLR2 stimulation was normal in the Hfe $\mathrm{KO}$ macrophages suggested that Mal/MyD88 signaling is intact in these cells. To address this issue further, we analyzed activation of the ERK and p38 kinases, an important Mal/MyD88-dependent response, in WT and Hfe KO macrophages following LPS stimulation. We found no obvious differences between the 2 types of cells with respect to the activation of either of the kinases (Figure 2). We also found no differences between WT and Hfe $\mathrm{KO}$ macrophages with respect to LPS-induced NF- $\kappa \mathrm{B}$ activation, as measured by IкB $\alpha$ degradation (Supplemental Figure 4). These observations provide further support for the idea that Hfe deficiency does not impair Mal/MyD88-dependent signaling.

TRAM/TRIF-dependent responses activated by TLR4 are impaired in Hfe KO macrophages. The normal Mal/MyD88-dependent responses in the Hfe KO macrophages raise the possibility that the attenuation of LPS-induced TNF- $\alpha$ expression in these cells may be caused by an abnormality of the TRAM/TRIF pathway, an idea supported by prior work showing that both TRAM and TRIF are required for the production of proinflammatory cytokines in response to TLR4 activation $(28,29)$. To address this possibility directly, we analyzed LPS-induced upregulation of IFN- $\beta$, a response that is mediated

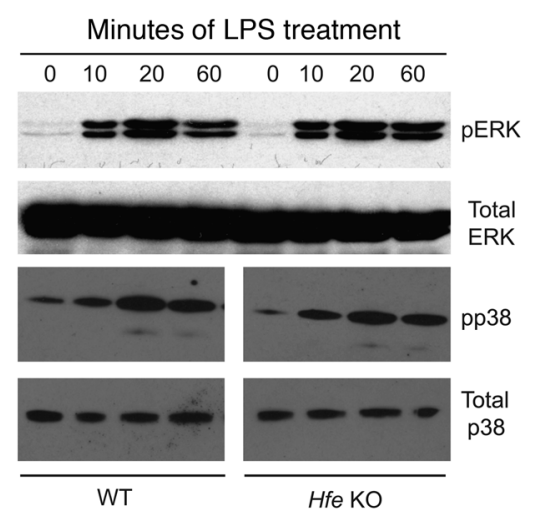

Figure 2

LPS-induced activation of ERK and p38 kinase pathways is intact in $\mathrm{Hfe} \mathrm{KO}$ macrophages. WT and Hfe KO macrophages were stimulated for the indicated times with $100 \mathrm{ng} / \mathrm{ml}$ LPS. Cell lysates were prepared, and Western blotting was performed to determine levels of total and phosphorylated kinases. Similar results were obtained in 2 separate experiments. 
A
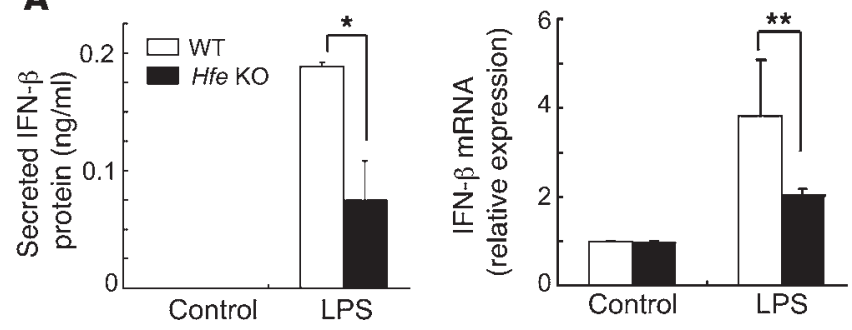

B
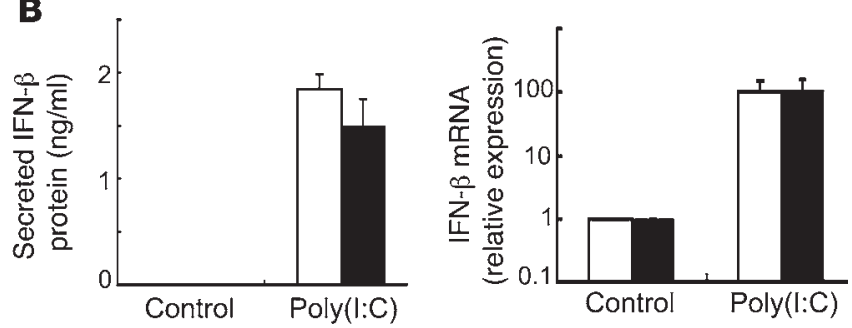

Figure 3

Induction of IFN- $\beta$ by activation of TLR4, but not TLR3, is abnormal in Hfe $\mathrm{KO}$ macrophages. WT and Hfe $\mathrm{KO}$ macrophages were stimulated with (A) $100 \mathrm{ng} / \mathrm{ml}$ LPS or (B) $10 \mu \mathrm{g} / \mathrm{ml}$ poly $(\mathrm{l}: \mathrm{C})$ for 6 hours $(n=3$ per group). Supernatants were collected and analyzed by ELISA for IFN- $\beta$. Data represent mean $\pm S D$. Similar results were obtained in 2 separate experiments. ${ }^{\star} P=0.026 ;{ }^{*} P=0.039$.

by TRAM/TRIF signals $(26,27)$. We found that the LPS-stimulated $\mathrm{Hfe} \mathrm{KO}$ macrophages expressed significantly lower levels of IFN- $\beta$ mRNA and protein than did WT cells (Figure 3A), which supports the idea that there is an impairment of TRAM/TRIF signaling in the mutant macrophages.

To further characterize the TRAM/TRIF signaling pathway in the Hfe KO macrophages, we examined the upregulation of IFN- $\beta$ after stimulation of TLR3 with the synthetic ligand poly(I:C). This response involves recruitment of TRIF and activation of signaling events downstream of this adaptor, but it does not require TRAM $(26,27)$. There was no significant difference between WT and $H f e$ $\mathrm{KO}$ macrophages in the poly(I:C)-induced expression of IFN- $\beta$ mRNA and protein (Figure 3B). This observation suggests that the functions of TRIF and signal transducing molecules distal to TRIF are unaffected by Hfe deficiency.

We previously showed that $\mathrm{Hfe} \mathrm{KO}$ macrophages have a reduction of free intracellular iron as a result of elevated FPN expression (25). To determine whether the abnormality of LPS-induced TRAM/ TRIF-dependent responses in the Hfe $\mathrm{KO}$ macrophages is related to the low levels of iron in these cells, we treated WT macrophages with the membrane-permeable iron chelator salicaldehyde isonicotinoyl hydrazone (SIH) in order to reduce the intracellular pool of free iron $(25,30)$. SIH treatment had a clear inhibitory effect on LPS-induced IFN- $\beta$ expression, but did not affect IFN- $\beta$ induction in response to poly(I:C) (Figure 4). Thus, lowering intracellular iron levels in WT macrophages resulted in an impairment of TLR4-activated, but not TLR3-activated, TRAM/TRIF-dependent responses, similar to the effects of Hfe deficiency. This finding provides further support for the idea that low intracellular iron contributes to the abnormal cytokine response of the Hfe KO macrophages.

Attenuated intestinal inflammation in WT mice after manipulation of iron homeostasis. Our results thus far indicated that Hfe deficiency and the associated reduction of intracellular iron in macrophages impairs TRAM/TRIF-dependent cytokine responses activated by TLR4. These findings raise the possibility that deliberate lowering of iron levels in macrophages may offer a way to suppress inflammation. To test this idea, we took the approach of inhibiting hepcidin expression in order to mimic the alterations in iron metabolism associated with $\mathrm{Hfe}$ deficiency. For this purpose, we used the small molecule inhibitor dorsomorphin, which has been shown recently to block the bone morphogenetic protein (BMP) receptor-activated signals required for hepcidin upregulation in response to inflammatory stimuli (31-33).

We found that oral infection of WT mice with S. typhimurium was associated with a significant increase in hepcidin expression in the liver (Figure 5A). Dorsomorphin treatment significantly inhibited the increase in liver hepcidin expression induced by Salmonella (Figure 5B) and also caused a partial reversal of the decrease in serum iron levels caused by the infection (Figure 5C). Most interestingly, dorsomorphin attenuated the severity of Salmonella-induced intestinal inflammation, as indicated by multiple observations. As shown in Figure 6A, Salmonella infection of the vehicle-treated mice led to obvious shrinkage and edema of the cecum, whereas these changes were less severe in the dorsomorphin-treated animals. In keeping with these differences, the cecal histopathology after Salmonella infection showed reduced inflammatory cell infiltration and more normal-appearing epithelial architecture in the dorsomorphin-treated mice compared with the vehicle-treated animals (Figure 6B). Finally, dorsomorphin treatment significantly lowered levels of TNF- $\alpha$ mRNA in the ceca of the Salmonella-infected mice (Figure 6C). Dorsomorphin treatment did not have significant effects on the tissue Salmonella burden, although the number of bacteria recovered from the liver in the dorsomorphin-treated group was more variable than in the vehicle-treated animals (Figure 6D). Thus, inhibition of hepcidin upregulation by dorsomorphin was associated with a reduction in the in vivo inflammatory response to Salmonella without a major influence on bacterial replication.

The effect of inhibiting hepcidin expression on inflammation was not confined to acute infectious enterocolitis. We tested the effect of an analog of dorsomorphin, LDN-193189, in a model of chronic colitis induced in $I l 10 \mathrm{KO}$ mice by administration of piroxicam (34). Piroxicam administration resulted in an increase in hepcidin
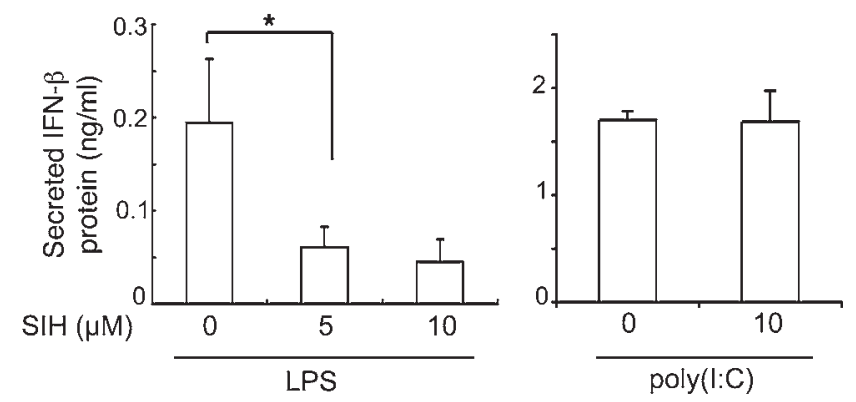

\section{Figure 4}

SIH inhibits LPS-induced, but not poly(l:C)-induced, expression of IFN- $\beta$ in WT macrophages. WT macrophages were stimulated with $100 \mathrm{ng} / \mathrm{ml}$

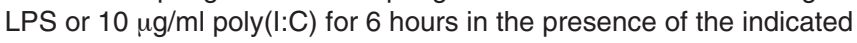
concentrations of SIH ( $n=3$ per group). Supernatants were collected and analyzed by ELISA for IFN- $\beta$. Data represent mean \pm SD. Similar results were obtained in 2 separate experiments. ${ }^{*} P=0.03$. 
A

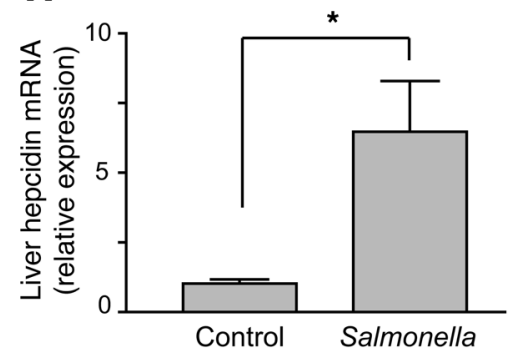

B

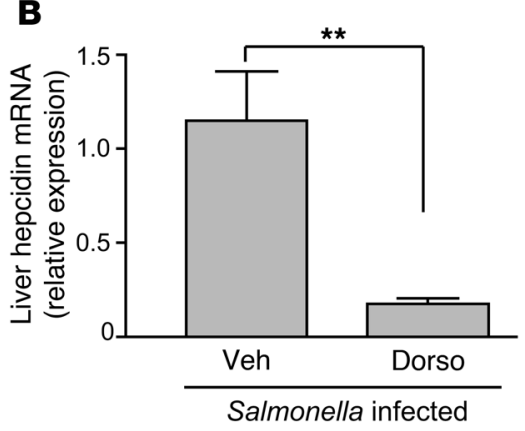

C

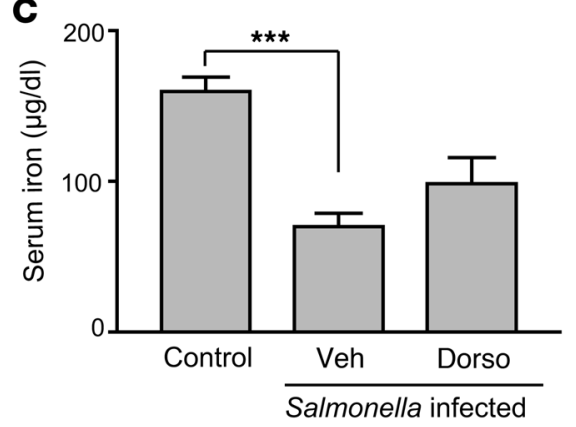

Figure 5

Effects of dorsomorphin on Salmonella-induced changes in hepcidin expression and serum iron levels. (A and B) Liver hepcidin mRNA was measured by quantitative RT-PCR in (A) control or Salmonella-infected WT mice 48 hours after infection $(n=5$ per group) and in (B) Salmonellainfected WT mice treated with vehicle $(n=7)$ or dorsomorphin $(n=6)$. (C) Serum iron levels were measured at the same time $(n=5$ [control]; 7 [vehicle]; 5 [dorsomorphin]). Data represent mean \pm SEM. ${ }^{\star} P=0.04 ;{ }^{\star \star} P=0.01 ;{ }^{\star \star \star} P<0.0001$.

expression, which was significantly inhibited by treatment with LDN-193189 (Figure 7A). Furthermore, treatment with the dorsomorphin analog resulted in a decrease in the severity of intestinal inflammation, as indicated by histopathology (data not shown) and by a significant reduction in colon IL-6 mRNA levels (Figure 7B).

Dorsomorphin and LDN-193189 are structurally related compounds that inhibit hepcidin expression by blocking BMP receptor signaling $(33,35)$. Therefore, it seemed possible that the influence of these compounds on intestinal inflammation could be related to an off-target effect common to both drugs and not to inhibition of hepcidin. To address this possibility, we examined the effect of a third and structurally distinct inhibitor of hepcidin expression, HJV.Fc, a soluble recombinant fusion protein that encodes the extracellular domain of hemojuvelin, a coreceptor for BMPs (32). HJV.Fc inhibits hepcidin expression by blocking the interaction of BMPs with their receptor rather than acting on intracellular signal transduction. The differences in structure and mode of action between HJV.Fc and the other hepcidin inhibitors made it unlikely that all 3 would have similar off-target effects that altered intestinal inflammation.

Freshly isolated peritoneal macrophages from WT mice injected in vivo with HJV.Fc produced significantly lower levels of TNF- $\alpha$ and IL- 6 in response to in vitro stimulation with LPS than did macrophages from vehicle-injected WT mice (Supplemental Figure 5 and data not shown), confirming the ability of this hepcidin-blocking reagent to inhibit macrophage cytokine responses. We then tested the effect of HJV.Fc in the piroxicam/Il10 KO colitis model. HJV.Fc treatment significantly inhibited the piroxicam-induced increase in hepcidin expression (Supplemental Figure 6A) and also reduced the severity of colitis as evaluated by histopathology (Supplemental Figure 6B) and tissue IL-17 mRNA levels (Supplemental

\section{Figure 6}

Effects of dorsomorphin treatment on Salmonella-induced intestinal inflammation in WT mice. (A-C) Groups of WT mice treated with vehicle or dorsomorphin were infected with Salmonella, and intestinal inflammation was evaluated 48 hours later by $(\mathbf{A})$ gross appearance of cecum, (B) cecal histopathology (original magnification, $\times 40$ ), and (C) cecal TNF- $\alpha$ mRNA levels (mean \pm SEM). (D) Numbers of Salmonella present in the liver were assessed at the same time; data points represent individual mice. $n=5$ (uninfected); 7 (vehicle); 6 (dorsomorphin). ${ }^{*} P=0.024$.
Figure 6C). Thus, blocking hepcidin upregulation with HJV.Fc was also associated with attenuation of intestinal inflammation.

\section{Discussion}

The results reported here demonstrate a surprising specificity for the effect of Hfe deficiency and the associated reduction of intramacrophage free iron levels on TLR-activated responses. The data indicate that the abnormality in TLR function in the Hfe KO macrophages not only is specific to TLR4, but also appears to affect only 1 of the 2 signaling pathways activated by this receptor, namely, that dependent on the TRAM and TRIF adaptor proteins.
A

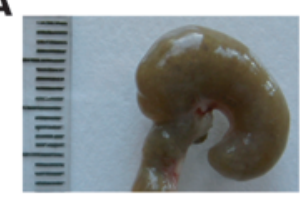

Uninfected
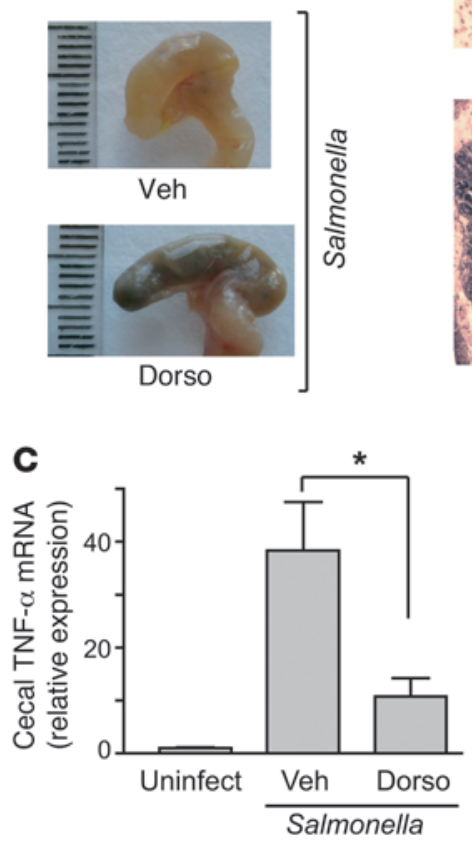

B

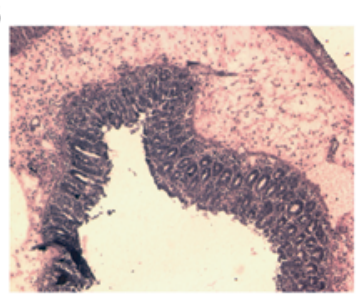

Veh

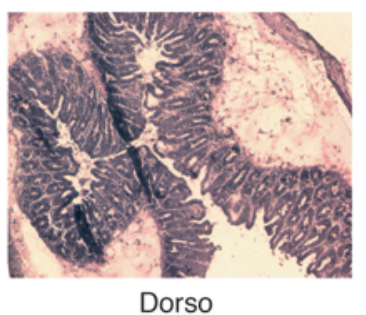

D

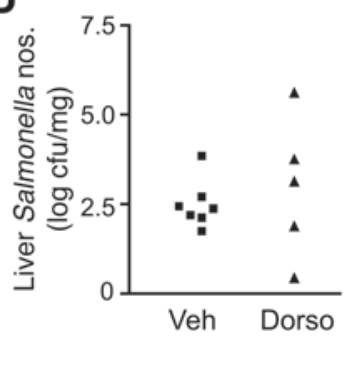


A
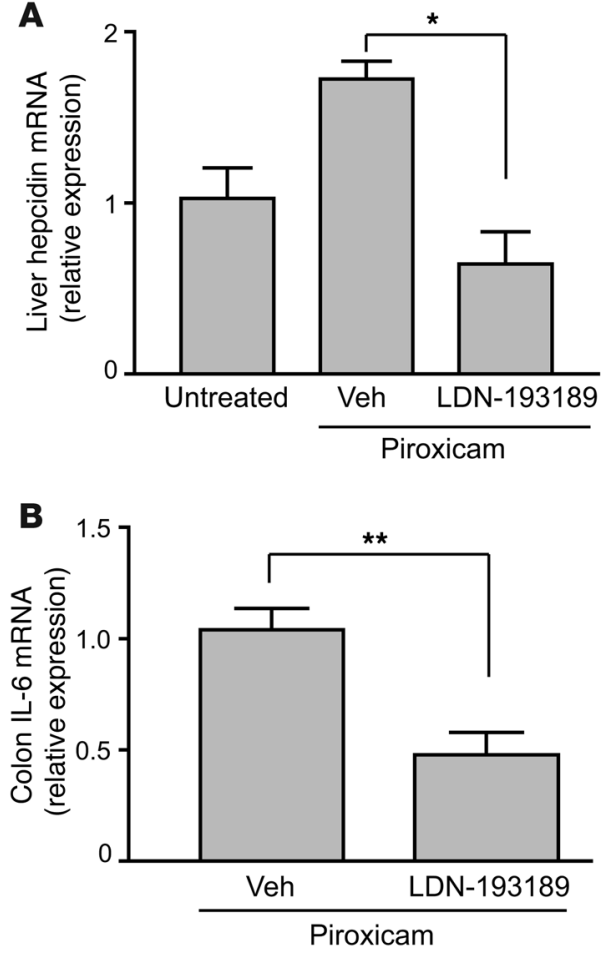

Furthermore, based on what is known about TLR signaling and on our finding that upregulation of IFN- $\beta$ in the Hfe KO macrophages was impaired in response to LPS but not poly(I:C), the simplest interpretation of the data is that $H f e$ deficiency and the associated decrease in intramacrophage iron levels negatively impact the MyD88-independent signaling pathway at a step unique to TLR4, that is, proximal to TRIF (Figure 8 ). Such an abnormality would also explain the reduction in Salmonella- and LPS-induced production of TNF- $\alpha$ and IL- 6 in the Hfe KO macrophages reported here and in our earlier work (25), because both TRAM and TRIF are required for expression of these cytokines $(28,29)$.

Among the TLRs, TLR4 is unique in being connected to both Mal/ MyD88 and TRAM/TRIF signaling pathways $(26,27)$. Upon activation by ligand, TLR 4 engages the 2 sets of adaptor proteins sequentially. Mal and MyD88 are recruited to the TLR4 cytoplasmic domain at the plasma membrane, but the interaction with TRAM and subsequently TRIF requires endocytosis of the receptor (36). Thus, there are several aspects of the TLR4-specific activation of TRIF-dependent signals that could be adversely affected by the low intramacrophage iron levels associated with $H f e$ deficiency, including TLR4 endocytosis, TLR4/TRAM interactions, TRAM expression, and TRAM/TRIF interactions. The list of possibilities is further extended by the fact that TRAM is known to undergo posttranslational myristoylation, a modification that is required for correct subcellular localization and function $(36,37)$. Although the Hfe KO macrophages had a modest reduction in cell surface TLR4 expression (Supplemental Figure $7 \mathrm{~A}$ ), we believe this is unlikely to be a major factor in the abnormal signaling, since activation of the ERK and p38 kinases was as robust as in the WT cells. Expression of the TRAM and TRIF adaptors also appeared to be normal in the $H f e \mathrm{KO}$ macrophages, at least at the mRNA level (Supplemental Figure 7B). Further work will be required to determine exactly how low intracellular iron inhibits TLR4-activated signal transduction via the TRAM/TRIF pathway.

\section{Figure 7}

LDN-193189 suppresses hepcidin upregulation and intestinal inflammation in piroxicam-induced colitis. $/ / 10 \mathrm{KO}$ mice were treated with piroxicam for 2 weeks and then injected with vehicle or LDN-193189 for an additional week. The animals were sacrificed, and (A) liver hepcidin mRNA ( $n=3$ [untreated and vehicle]; 4 [LDN-193189]) and (B) colon IL-6 mRNA ( $n=9$ [vehicle]; 12 [LDN-193189]) were measured by quantitative RT-PCR. Data represent mean \pm SEM. ${ }^{\star} P=0.005 ;{ }^{* *} P=0.0007$.

Regardless of the precise mechanism of action, it is clear that the low intramacrophage iron levels associated with Hfe deficiency have substantial impact on the TLR4-induced production of various inflammatory cytokines. Furthermore, the results of our experiments with the inhibitors of hepcidin expression indicate that the acute, hepcidin-mediated changes in iron homeostasis that occur during infection can also influence inflammatory responses. Specifically, the increase in intramacrophage iron caused by hepcidin-induced FPN downregulation could be considered to act as a proinflammatory signal that facilitates expression of cytokines such as TNF- $\alpha$. This idea provides a teleologic explanation for the increase in hepcidin stimulated by inflammatory signals: the response may have protective value in terms of ramping up macrophage activation. Our findings also raise the possibility that blocking hepcidin expression may represent a novel approach to controlling inflammation. Dorsomorphin, LDN-193189, and HJV.Fc treatment reduced the severity of intestinal inflammation in models of acute infectious colitis and chronic noninfectious colitis. Although we cannot definitively exclude the possibility that the attenuation of inflammation represents the outcome of effects other than those on hepcidin expression, we think it is unlikely in the context of our observations with the $\mathrm{Hfe} \mathrm{KO}$ mice, and given that HJV.Fc and the other 2 inhibitors are structurally distinct and inhibit BMP-mediated hepcidin expression by quite different mechanisms $(32,33,35)$.

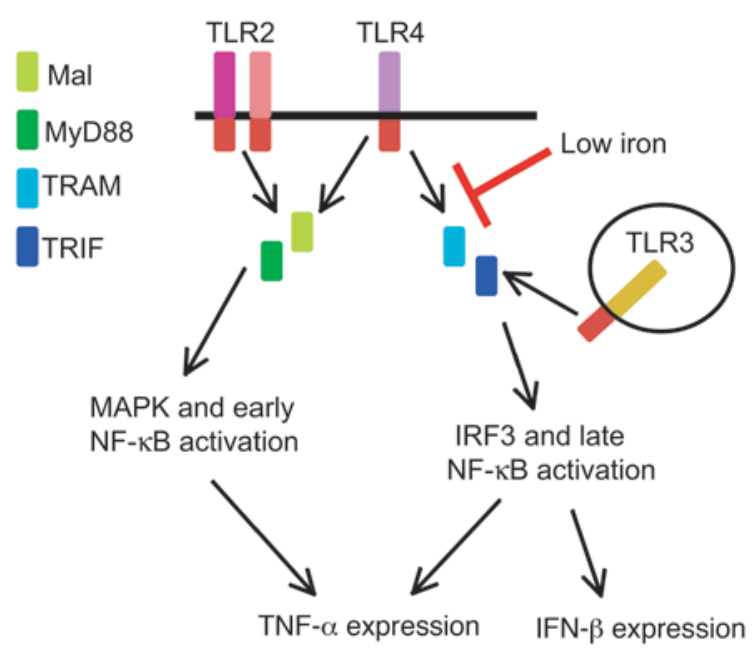

Figure 8

Influence of low intracellular iron on LPS-induced cytokine expression. Based on the effects of Hfe deficiency and iron chelation on responses to TLR2, TLR3, and TLR4 stimulation, low intracellular iron is likely to impair TLR4 signaling at a step in the TRAM/TRIF pathway proximal to TRIF. IRF3, IFN regulatory factor 3 . 
Iron chelation therapy with agents such as desferrioxamine has been tried previously for chronic inflammatory conditions, such as rheumatoid arthritis, and limited success has been reported in some studies (38). Inhibition of hepcidin expression or function could have advantages over the nonspecific reduction of iron produced by chelators since its effects would be confined largely to FPN-expressing cells such as macrophages. In addition, the increase in FPN expression that would follow reduced circulating hepcidin levels would increase iron availability and help to correct the anemia associated with many longstanding inflammatory states (39). Our results suggest that this strategy may be worth further investigation.

The findings presented here, as well those we have reported previously (25), strongly support the idea that low intracellular iron levels play an important role in the abnormal TLR4 signaling and cytokine response of the $\mathrm{Hfe} \mathrm{KO}$ macrophages. However, it has been shown recently that the hepcidin-FPN interaction leads to activation of the Jak2 kinase (40). This study implicated Jak2dependent phosphorylation of FPN in downregulation of the receptor, but it is also possible that downstream signals activated by Jak 2 could influence gene expression and, therefore, that deficiency of such signals could contribute to the abnormal cytokine production by macrophages from Hfe KO mice. This is a possibility that deserves exploration.

In conclusion, we have shown that Hfe deficiency and the associated decrease in intramacrophage iron have a negative influence on signaling through the TRAM/TRIF pathway activated by TLR4, probably at a step proximal to TRIF. Our findings reveal what we believe to be a novel, iron-dependent mechanism that modulates TLR4 signals; moreover, they provide an explanation for the attenuated in vitro and in vivo inflammatory responses of Hfe KO mice (25) and, potentially, for the increased susceptibility of individuals with hemochromatosis to certain bacterial infections (22-24). We have also shown that manipulation of iron homeostasis may represent a novel strategy for controlling inflammation. Further investigation of the interactions between iron metabolism and inflammatory responses will allow for refinement of this therapeutic approach.

\section{Methods}

Animals. WT C57BL/6 mice were obtained from the Jackson Laboratory. $\mathrm{Hfe} \mathrm{KO}$ mice on the C57BL/6 background were originally provided by N.C. Andrews (Duke University, Durham, North Carolina, USA; ref. 20). All mice were bred and housed in a specific pathogen-free facility at Massachusetts General Hospital. Animals were given water and standard laboratory chow ad libitum and used at 7-12 weeks of age. All animal experiments were approved by the Massachusetts General Hospital Subcommittee on Research Animal Care.

Macrophage stimulation. Thioglycollate-elicited peritoneal macrophages were prepared as previously described $(25,41)$ and stimulated with $100 \mathrm{ng} /$ $\mathrm{ml}$ LPS (Ultra-pure; List Biological Laboratories), $1 \mu \mathrm{g} / \mathrm{ml}$ of the synthetic bacterial lipopeptide Pam3CSK4, or $10 \mu \mathrm{g} / \mathrm{ml}$ poly(I:C) (both from Invivogen) for the indicated times in triplicate wells of a 24-well tissue culture plate. SIH was provided by P. Ponka (McGill University, Montreal, Quebec, Canada) and was added to macrophage cultures 30 minutes before addition of TLR ligands. Cell supernatants were analyzed by ELISA for various cytokines using specific antibody pairs obtained from R\&D Systems or BD Biosciences - Pharmingen. Total RNA was prepared from the cells using TRIzoL reagent (Invitrogen), and quantitative RT-PCR was carried out to determine levels of cytokine mRNA according to previously described methods $(25,41)$. Primers used for amplification of TNF- $\alpha$, IL- 6 , and the housekeeping transcript 36B4 have been described previously (25). Other primers used were as follows: IFN- $\beta$ sense, $5^{\prime}$-CAGCTCCAAGAAAGGACGAAC- ${ }^{\prime}$; IFN- $\beta$ antisense, $5^{\prime}$-GGCAGTGTAACTCTTCTGCAT- 3 '; TRAM sense, 5'-CGATCAAGACGGCCATGAGTC-3'; TRAM antisense, 5'-CTCGTCGGTGTCATCTTCTGC-3'; TRIF sense, $5^{\prime}$-AACCTCCACATCCCCTGTTTT-3'; TRIF antisense, 5'-GCCCTGGCATGGATAACCA-3'. Immunoblotting procedures used for analysis of MAP kinase activation and IкB $\alpha$ degradation have been described in detail elsewhere (41). Bone marrow-derived macrophages were prepared by growing bone marrow cells in medium containing 10\% L-929-conditioned medium for 7 days. The cells were then stimulated and analyzed as described above.

Flow cytometry. Thioglycollate-elicited peritoneal macrophages were stained with a phycoerythrin-conjugated anti-TLR4 antibody (clone UT41; eBioscience) and subjected to flow cytometry on a FACScan (BD) as previously described (42). The assay for intracellular iron based on iron-dependent quenching of calcein fluorescence has been described previously (25).

Salmonella-induced enterocolitis. Following the protocol described by Barthel et al. (43), mice were given a single $20-\mathrm{mg}$ oral dose of streptomycin followed 24 hours later by oral infection with $10^{8} \mathrm{cfu}$ of the streptomycin-resistant, WT, invasion-competent SL1344 strain of S. typhimurium. Treatment with dorsomorphin $(12.5 \mu \mathrm{g} / \mathrm{g}$ BW i.p.; Calbiochem) or an equivalent volume of the vehicle DMSO was started at the same time as the streptomycin was administered and was continued twice daily for the duration of the experiment. Animals were sacrificed 48 hours after infection. At necropsy, intestinal inflammation was assessed by gross appearance of the cecum, cecal histopathology, and quantitative RT-PCR-based measurement of cecal TNF- $\alpha$, all as previously described in detail (25). Liver hepcidin expression was determined by quantitative RT-PCR with the following primers: sense, 5'-AGAGCTGCAGCCTTTGCAC-3', antisense, 5'-GAAGATGCAGATGGGGAAGT-3'. Bacterial burden was assessed by homogenizing weighed portions of liver in sterile $1 \%$ Triton $\mathrm{X}-100$ and plating serial dilutions of the homogenates on LB agar containing $50 \mu \mathrm{g} / \mathrm{ml}$ streptomycin.

Piroxicam-induced colitis in Il10 KO mice. The protocol described by Berg et al. was followed (34). In brief, 4- to 6-week-old Il10 KO mice (C57BL/6 background, breeders obtained from Jackson Laboratory) were fed with $60 \mathrm{mg}$ piroxicam (Sigma-Aldrich) mixed with $250 \mathrm{~g}$ NIH-31M chow for 1 week, followed by $80 \mathrm{mg}$ piroxicam plus $250 \mathrm{~g}$ chow for an additional 1 week. The animals were then placed on their regular diet and treated with either LDN-193189 or HJV.Fc over the course of the following week. LDN193189 was custom synthesized, as described by Cuny et al. (35), by Shanghai United Pharmatec and dissolved at a concentration of $0.25 \mathrm{mg} / \mathrm{ml}$ in $2 \% 2$-hydroxypropyl- $\beta$-cyclodextrin. Mice were injected i.p. twice daily with $3 \mathrm{mg} / \mathrm{kg} \mathrm{BW}$ LDN-193189 or with an equivalent volume of the vehicle. HJV.Fc (prepared as described in ref. 32) was injected i.p. 3 times over the course of a week at a dose of $15 \mathrm{mg} / \mathrm{kg} \mathrm{BW}$. Control animals were injected with a corresponding volume of PBS vehicle. The animals were sacrificed, and colonic inflammation was evaluated by histopathology. An investigator blinded to the identity of the samples examined the hematoxylin and eosin-stained sections and graded the severity of inflammation based on a previously described scoring system (44). Liver hepcidin expression was determined by quantitative RT-PCR at the same time.

Statistics. Results were compared by 2-tailed Student's $t$ test or MannWhitney $\mathrm{U}$ test. A $P$ value less than 0.05 was considered significant. $n$ refers to number of individual mice or stimulations.

\section{Acknowledgments}

This work was supported by funds from the Broad Medical Research Program (grant no. IBD-0253) to Bobby J. Cherayil and from Wyeth 
Nutrition to L. Wang. C.C. Hong received support from the Center for Research in Fibrodysplasia Ossificans Progressiva and Related Disorders. H.Y. Lin was supported in part by grant R01-071837 from the National Institute of Diabetes and Digestive and Kidney Diseases, NIH. We are grateful to Nancy Andrews for providing the $\mathrm{Hfe} \mathrm{KO}$ mice and to Prem Ponka for the gift of SIH. We thank Sarah A. Faasse for technical assistance with iron assays.

1. Schaible, U.E., and Kaufmann, S.H. 2004. Iron and microbial infection. Nat. Rev. Microbiol. 2:946-953.

2. Gangaidzo, I.T., et al. 2001. Association of pulmonary tuberculosis with increased dietary iron. J. Infect. Dis. 184:936-939.

3. Gordeuk, V.R., McLaren, C.E., MacPhail, A.P., Deichsel, G., and Bothwell, T.H. 1996. Associations of iron overload in Africa with hepatocellular carcinoma and tuberculosis: Strachan's 1929 thesis revisited. Blood. 87:3470-3476.

4. Magnus, S.A., Hambleton, I.R., Moosdeen, F., and Serjeant, G.R. 1999. Recurrent infections in homozygous sickle cell disease. Arch. Dis. Child. 80:537-541.

5. Moyo, V.M., Gangaidzo, I.T., Gordeuk, V.R., Kiire, C.F., and Macphail, A.P. 1997. Tuberculosis and iron overload in Africa: a review. Cent. Afr. J. Med. 43:334-339.

6. Wanachiwanawin, W. 2000. Infections in E-beta thalassemia. J. Pediatr. Hematol. Oncol. 22:581-587.

7. Murray, M.J., Murray, A.B., Murray, M.B., and Murray, C.J. 1978. The adverse effect of iron repletion on the course of certain infections. Br. Med. J. 2:1113-1115.

8. Nyakeriga, A.M., et al. 2004. Iron deficiency and malaria among children living on the coast of Kenya. J. Infect. Dis. 190:439-447.

9. Sazawal, S., et al. 2006. Effects of routine prophylactic supplementation with iron and folic acid on admission to hospital and mortality in preschool children in a high malaria transmission setting: community-based, randomized, placebo-controlled trial. Lancet. 367:133-143.

10. Puschmann, M., and Ganzoni, A.M. 1977. Increased resistance of iron-deficient mice to Salmonella infection. Infect. Immun. 17:663-664.

11. Wang, L., and Cherayil, B.J. 2009. Ironing out the wrinkles in host defense: interactions between iron homeostasis and innate immunity. J. Innate Immun. 1:455-464.

12. Pietrangelo, A. 2006. Hereditary hemochromatosis. Annu. Rev. Nutr. 26:251-270.

13. Feder, J.N., et al. 1996. A novel MHC class I-like gene is mutated in patients with hereditary haemochromatosis. Nat. Genet. 13:399-408.

14. Andrews, N.C. 2007. Iron homeostasis. Annu. Rev. Physiol. 69:69-85.

15. Nemeth, E., and Ganz, T. 2006. Regulation of iron metabolism by hepcidin. Annu. Rev. Nutr. 26:323-342.

16. Goswami, T., and Andrews, N.C. 2006. Hereditary hemochromatosis protein, HFE, interaction with
Received for publication May 21, 2009, and accepted in revised form August 19, 2009.

Address correspondence to: Bobby J. Cherayil, Mucosal Immunology Laboratory, Massachusetts General Hospital, Building 114, $16^{\text {th }}$ Street, Charlestown, Massachusetts 02129, USA. Phone: (617) 7264170; Fax: (617) 726-4172; E-mail: cherayil@helix.mgh.harvard.edu. transferrin receptor 2 suggests a molecular mechanism for mammalian iron sensing. J. Biol. Chem. 281:28494-28498.

17. Nemeth, E., et al. 2004. Hepcidin regulates cellular iron efflux by binding ferroportin and inducing its degradation. Science. 306:2090-2093.

18. Ahmad, K.A., et al. 2002. Decreased liver hepcidin expression in the Hfe knockout mouse. Blood Cells Mol. Dis. 29:361-366.

19. Bahram, S., et al. 1999. Experimental hemochromatosis due to MHC class I HFE deficiency: immune status and iron metabolism. Proc. Natl. Acad. Sci. U. S. A. 96:13312-13317.

20. Levy, J.E., Montross, L.K., Cohen, D.E., Fleming, M.D., and Andrews, N.C. 1999. The C282Y mutation causing hereditary hemochromatosis does not produce a null allele. Blood. 94:9-11.

21. Zhou, X.Y., et al. 1998. HFE gene knock-out produces a mouse model of hereditary hemochromatosis. Proc. Natl. Acad. Sci. U. S. A. 95:2492-2497.

22. Bullen, J.J., Spalding, P.B., Ward, C.G., and Gutteridge, J.M. 1991. Hemochromatosis, iron and septicemia caused by Vibrio vulnificus. Arch. Intern. Med. 151:1606-1609.

23. Doherty, C.P. 2007. Host-pathogen interactions: the role of iron. J. Nutr. 137:1341-1344.

24. Gerhard, G.S., et al. 2001. Vibrio vulnificus septicemia in a patient with the hemochromatosis HFE C282Y mutation. Arch. Pathol. Lab. Med. 125:1107-1109.

25. Wang, L., et al. 2008. Attenuated inflammatory responses in hemochromatosis reveal a role for iron in the regulation of macrophage cytokine translation. J. Immunol. 181:2723-2731.

26. O'Neill, L.A. 2008. The interleukin-1/Toll-like receptor superfamily: 10 years of progress. Immunol. Rev. 226:10-18.

27. Kawai, T., and Akira, S. 2008. Toll-like receptor and RIG-I receptor signaling. Ann. N. Y. Acad. Sci. 1143:1-20.

28. Yamamoto, M., et al. 2003. Role of adaptor TRIF in the MyD88-independent Toll-like receptor signaling pathway. Science. 301:640-643.

29. Yamamoto, M., et al. 2003. TRAM is specifically involved in the Toll-like receptor 4-mediated MyD88-independent signaling pathway. Nat. Immunol. 4:1144-1150.

30. Simunek, T., et al. 2005. SIH - a novel lipophilic iron chelator - protects $\mathrm{H} 9 \mathrm{c} 2$ cardiomyoblasts from oxidative stress-induced mitochondrial injury and cell death. J. Mol. Cell. Cardiol. 39:345-354.

31. Babitt, J.L., et al. 2006. Bone morphogenetic protein signaling by hemojuvelin regulates hepcidin expression. Nat. Genet. 38:531-539.

32. Babitt, J.L., et al. 2007. Modulation of bone morphogenetic protein signaling in vivo regulates systemic iron balance. J. Clin. Invest. 117:1933-1939.

33. Yu, P.B., et al. 2008. Dorsomorphin inhibits BMP signals required for embyrogenesis and iron metabolism. Nat. Chem. Biol. 4:33-41.

34. Berg, D.J., et al. 2002. Rapid development of colitis in NSAID-treated IL10-deficient mice. Gastroenterology. 123:1527-1542.

35. Cuny, G.D., et al. 2008. Structure-activity relationship study of bone morphogenetic protein signaling inhibitors. Bioorg. Med. Chem. Lett. 18:4388-4392.

36. Kagan, J.C., et al. 2008. TRAM couples endocytosis of Toll-like receptor 4 to the induction of interferon-beta. Nat. Immunol. 9:361-368.

37. Rowe, D.C., et al. 2006. The myristoylation of TRIFrelated adaptor molecule is essential for Toll-like receptor 4 signal transduction. Proc. Natl. Acad. Sci. U. S. A. 103:6299-6304.

38. Magaro, M., et al. 1990. Iron chelation in rheumatoid arthritis: clinical and laboratory evaluation. Ann. Rheum. Dis. 49:268-269.

39. Weinstein, D.A., et al. 2002. Inappropriate expression of hepcidin is associated with iron refractory anemia: implications for the anemia of chronic disease. Blood. 100:3776-3781.

40. De Domenico, I., Lo, E., Ward, D.M., and Kaplan, J. 2009. Hepcidin-induced internalization of ferroportin requires binding and cooperative interaction with Jak2. Proc. Natl. Acad. Sci. U. S. A. 106:3800-3805.

41. Li, Q., and Cherayil, B.J. 2003. Role of Toll-like receptor 4 in macrophage activation and tolerance during Salmonella enterica serovar Typhimurium infection. Infect. Immun. 71:4873-4882.

42. Rhee, S.J., Walker, W.A., and Cherayil, B.J. 2005. Developmentally regulated intestinal expression of IFN $\gamma$ and its target genes and the age-specific response to enteric Salmonella infection. J. Immunol. 175:1127-1136.

43. Barthel, M., et al. 2003. Pretreatment of mice with streptomycin provides a Salmonella enterica serovar Typhimurium colitis model that allows analysis of both pathogen and host. Infect. Immun. 71:2839-2858.

44. Chen, C.C., Louie, S., McCormick, B., Walker, W.A., and Shi, H.N. 2005. Concurrent infection with an intestinal helminth parasite impairs host resistance to enteric Citrobacter rodentium and enhances Citrobacter-induced colitis in mice. Infect. Immun. 73:5468-5481. 